Section 1.

CELESTIAL REFERENCE SYSTEM AND FRAME 


\title{
Celestial Reference Systems - An Overview
}

\author{
J. Kovalevsky \\ CERGA, Observatoire de la Côte d'Azur, 06130 Grasse, France
}

\begin{abstract}
The paper starts with a short presentation of the conceptual foundations of celestial reference systems and of the IAU decisions taken in 1991. The necessity to define and use them in the framework of General Relativity induces some difficulties that are analyzed. In the case of kinematically defined reference systems, the origins of a possible residual rotation of the frames is analyzed. The same analysis holds also for an isolated dynamical reference system, for which further developments of the metric are necessary. Finally, a few topics concerning the non-isolated case of the barycentric reference system, the transformation between reference systems, and the significance of measurements are mentioned.
\end{abstract}

\section{Introduction}

This presentation is devoted only to celestial reference systems and not to reference frames which are realizations (or materializations) of a reference system. The latter is essentially a two step theoretical construction: one may distinguish an ideal reference system in which mathematical and physical properties are defined, and a conventional reference system in which the material model from which the frames are to be constructed is specified (Kovalevsky and Mueller, 1989). In what follows, I shall essentially deal with conventional celestial reference systems, the basics of the ideal system being described in this volume by Soffel and Klioner. We shall see that, even at this very preliminary stage of preparing the construction of a reference frame, the problems become quite involved if one takes into account the increasing accuracy of the observations.

Originally, a celestial reference system was simply defined as a system of coordinate axes with respect to which the positions of celestial bodies were referred. The underlying assumption was that space was an absolute Euclidean space and the time was absolute in the sense that it did not depend on the physical or dynamical state of the clock or of its position in space. The consequence was that the system did not change when translated for instance from the geocenter to the barycenter of the solar system, and a strict synchronization of clocks was always possible. To transform positions from a geocentric to a barycentric celestial system, it was sufficient to apply geometric (parallactic) and optical (aberration) corrections. In this space, a coordinate system that would be non-rotating with respect to distant extragalactic objects, would also ${ }^{2} \mathrm{e}$ dynamically inertial in the sense that the motions in the solar system could be represented without introducing Coriolis or centrifugal accelerations. 
Now, this Euclidean model had to be replaced by the relativistic model in which the space and the time are associated in a single geometrical object whose properties are defined by the distribution of matter in space. The space-time properties are formalized by a metric whose form is conventionally fixed. So, in 1991, the IAU has decided to set it in the following form:

$$
\mathrm{d} s^{2}=-c^{2} \mathrm{~d} \tau^{2}=-\left(1-2 w / c^{2}\right)\left(\mathrm{d} x^{0}\right)^{2}+\left(1+2 w / c^{2}\right)\left(\left(\mathrm{d} x^{1}\right)^{2}+\left(\mathrm{d} x^{2}\right)^{2}+\left(\mathrm{d} x^{3}\right)^{2}\right),
$$

where $c$ is the velocity of light, $\tau$ the proper time, $x^{0}$ is the time coordinate $\left(t=x^{0} / c\right.$ is called the coordinate time), and

$$
w=w_{m}+w_{e}
$$

is the sum of the Newtonian gravitational potential $w_{m}$ of the ensemble of masses, and of the tidal potential $w_{e}$ generated by bodies external to this ensemble, the latter potential vanishing at the chosen origin. This means in particular that a reference system is defined locally by specifying what is its center.

Another general way to express this is to present the four-dimensional metric tensor $g_{i, j}$ with $i$ and $j$ taking all values from 0 to 3 . Then, (1) is equivalent to:

$$
\begin{gathered}
g_{00}=\left(-1+2 w / c^{2}\right) \\
g_{i i}=\left(1+2 w / c^{2}\right) \\
g_{0 j}=0 \quad g_{i j}=0
\end{gathered}
$$

Formulas (1) to (3) are just the first terms of a development in power series in $c^{-1}$ of the elements of the complete metric tensor. They are sufficient in practically all the cases at the level of accuracy that was reached in 1991.

In a relativistic context, the word inertial has no meaning and, due to the curvature of space, it is not possible to define in the usual way the direction of an object in space. The consequence is that there are two types of difficulties. They can be overcome locally assuming low gravitation, with an approximation that depends on the completeness of the description of the mass distribution and on the modelling of the space-time metric. Formula (1) is the simplest of such models.

Conceptually, one may set and solve the various dynamical and kinematical problems in the framework of Einstein's theory of gravity in a very general fashion which is coordinate free in a purely geometrical approach. But the mathematical difficulties encountered have by far not yet been solved, and it is necessary to select a specific type of coordinate system. This usually means that one must impose a gauge condition. Let us note that the particular choice of the metic tensor (3) had the advantage that its simplicity allowed us to use any type of coordinates such as a harmonic coordinate system, or the parametrized post-Newtonian approximation.

\section{Kinematically defined reference systems}

The astrometric observation of extragalactic objects provides the apparent direction from which the light reaches the instrument. Let us forget the refraction 
problems due to the atmosphere or to the interplanetary, interstellar or intergalactic matter. The transport of the directions observed into the barycentric reference system is a problem that was globally solved in a very general case by Brumberg and Kopejkin (1989). However, in practice, there is still a strong traditional trend to treat separately the various effects (aberration, geodetic precession and light deflection), for instance as described in a simplified linear case by Green (1985), rather than globally.

The light describes a geodetic line in the curved space between the source and the barycenter. Within the solar system, it is variable with time and must be described from the known positions of masses. It is conventionally assumed that the kinematically defined reference system is such that the directions correspond to the directions from which the light arrives when it reaches the outskirts of the solar system. In other terms, they correspond to what they would be if all the masses within the solar system would have vanished. The assumption is that the solar system is isolated. It is somewhat similar to the non-relativistic reference system as previously defined. Locally, the reference system coincides with the Euclidean tangent space around the barycenter and can be used as such, noting however that the directions are not the physical directions projected on this space.

The theoretical rigorous background of this definition of a kinematically non-rotating celestial reference system is that it is an asymptotically flat spacetime (Minkowskian). The details can be found in Soffel (1989). It is based on the notion of Weyl parallelism in such a space which justifies the above approach, provided that the spatial axes are defined far away from material sources. However, in reality, the directions so defined do not trace the actual directions of objects, because the light undergoes numerous bendings while crossing the Galaxy and the extragalactic space.

Finally, the conventional definition rests on the physical validity of the postulate following which a set of remote extragalactic sources has no residual rotation. This is an astronomical as well as a cosmological problem. This is true at the millisecond-of-arc level of accuracy for which it is expected that, at the microsecond of arc level, the relative proper motions become observable. At a distance of $10^{9}$ light years, a relative motion of $1500 \mathrm{~km} \mathrm{~s}^{-1}$ produces an angular proper motion of $1 \mu$ as per year, a kind of precision that is not far from being within the reach of future space astrometry. One should also mention the instability of the radio-sources in their shapes and positions, and the variability of the emission distribution with time. This is a problem that concerns the construction of reference frames. If, as it is expected, the radio-sources are changing so that it is not possible to guarantee a few microacrsecond stability of the frame, the conventional definition of the international celestial reference system should be changed by shifting to optical extragalactic objects (which are more stable in position and luminosity) as soon as they will be observed with high accuracies by SIM or GAIA. In all cases, if the position shifts are randomly distributed, they will be treated as white noise. If they seem to produce an apparent bulk rotation, one may apply the inverse rotation to cancel it.

There is at least one effect that may resemble a global rotation or a zonal distortion if it is globally treated as such rather than properly corrected for: it is the variable part of aberration due to the motion of the Sun around the center of the Galaxy. At microsecond-of-arc accuracies, the effect is not negligible 
(Klioner and Soffel, 1998). Let us assume, as a first approximation, that one may represent the motion of the Sun as a circular orbit around the center of the Galaxy. Let $n$ be its yearly mean angular motion and $k=n / c$ the corresponding constant of aberration. The expression of the aberration effect in galactic coordinates is

$$
\begin{gathered}
\Delta l(t)=-k / \cos b \cos l(t), \\
\Delta b(t)=-k \sin b \sin l(t) .
\end{gathered}
$$

The constant part of this effect is not observable since if corresponds to a constant displacement of the coordinates of the extragalactic object and does not affect the the quality of the reference frame. But due to the assumed circular motion of the Sun, the galactic longitude has a yearly variation of $d l / d t=n$ The variable part of the galactic aberration is obtained by differentiating the above expressions with respect to $t$. One gets:

$$
\begin{gathered}
\delta(\Delta l(t))=k n / \cos b \sin l(t), \\
\delta(\Delta b(t))=-k n \sin b \cos l(t) .
\end{gathered}
$$

With the presently available estimations of the velocity of the Sun and its distance to the center of the Galaxy, the coefficient $k n$ is between 4 and $5 \mu$ as per year.

In fact, the actual reality of a bulk rotation of a system that is supposed to represent the Universe is questionnable. On one side, one may doubt that it is possible to find an intrinsic non-rotating physical system to which one would refer a large part of the Universe. On the other side, if we believe that Mach's principle is valid, (see, for instance, Rindler, 1977), then is this quest actually significant? For these reasons, the present definition of the international kinematic reference frame is quite satisfactory.

\section{Dynamically defined isolated reference systems}

In the past, in a non-relativistic approach, the celestial reference system was dynamically defined by setting the condition that the motion in the solar system is represented by a solution of a system of differential equations derived from Newton's law written in a fixed non-rotating system of coordinates, that is in the absence of Coriolis or centrifugal accelerations. In addition, it was supposed that it was isolated in the sense that any potential external to the system could be neglected.

The same assumptions can be extended in the framework of the theory of general relativity. The absence of Coriolis accelerations implies that there are no Coriolis terms in $g_{0 j}$. The isolation condition means that all galactic and extragalectic effects are neglected so that in formula (2) one has $w_{e}=$ 0 . The consequence of the absence of matter outside some volume is that the space-time is asymptotically flat (or Minkowskian) as already assumed for the kinematic reference frames. The spatial orientation of the reference system may then be chosen in such a way that it is fixed with respect to asymptotical flat space. In particular, if the reference system in the Minkowskian space is defined by the positions of extragalactic objects as presented in Section 2, then the 
isolated dynamical reference system is identical to the kinematic one, and there is no rotation between the two reference systems. If a rotation between the two systems is observed, it may mean either that the assumption $w_{e}=0$ is not valid or that the modelling of the mass distribution in the solar system is not adequate.

As already mentioned, the metric (1) was chosen by the IAU as the simplest possible. The increase of the accuracy of position and time observations leads us now to consider that the expressions (3) should be extended to higher order terms in $c^{-1}$. In particular, the present requirements are more demanding for time than for positions. It is suggested to limit the developments to the order of $c^{-3}$ for positions and $c^{-5}$ for time transformations. This would give the following (Soffel et al., 2000):

$$
\begin{gathered}
g_{00}=\left(-1+2 w / c^{2}\right)-2 w^{2} / c^{4}+\mathrm{O}\left(c^{-6}\right), \\
g_{i i}=\left(1+2 w / c^{2}\right)+\mathrm{O}\left(c^{-4}\right), \\
g_{0 j}=-4 w_{i} / c^{3}+\mathrm{O}\left(c^{-5}\right), \\
g_{i j}=0+\mathrm{O}\left(c^{-4}\right) .
\end{gathered}
$$

In these expressions, $w$ is no longer the Newtonian, but the relativistic potential and $w_{i}$ is the vector potential of the gravity related, for instance, with the LenseThirring acceleration due to gravito-magnetic effects of a rotating body (see for instance, Soffel, 1989, p. 95). The formulas (4) are proposed for adoption by the IAU.

A more complete expression, including additional relativistic terms in computing the potential can be found in Brumberg (1991a, p. 53). The terms neglected in formulas (4) are considered as negligible for the time being. The addition of higher order terms to formulas (3) increases drastically the difficulties of the relativistic treatment of problems. Not only one must take into account more terms and use non-Newtonian expressions for $w$, but the linearity of the equations in $c^{-2}$ disappear. The primary consequence of the non-linearity is that it is necessary to define the type of coordinates in which one works, that is to set a gauge condition. The PPN formulation, essentially linear, cannot be extended to a more complete metric and it will have to be abandoned whenever such high-order effects are necessary.

The most studied coordinates as yet in theoretical work are the harmonic ones. In addition, it was found to be a simplifying gauge for many kinds of applications (Soffel et al., 2000) and is used in the DE/LE series by JPL. The IAU is being proposed to impose it. This would be a good move, because it will lead to a unified approach of studies and avoid unnecessary tedious work in comparing the formulations obtained by different gauge conditions.

\section{Non-isolated dynamical reference systems}

Let us now assume that the reference system is not isolated. In this case, external potentials exist, so that $w_{e}$ does not vanish. In particular, one cannot any more use the the assumption that the coordinate system is in the asymptotic flat Minkowskian space-time unless the Galaxy is included in the internal space. 
This would lead us to define a galactocentric dynamical reference system, a possibility that cannot be envisaged with our present very poor knowledge of the dynamical properties of the Galaxy. In practice, then, one is condemned to model the barycentric external field. Actually, the effect is small and one can, in a first approximation, model the galactic potential simply by assuming a central point-like mass.

\section{Transformations between systems}

In astronomy, one must use at least two celestial reference systems: the barycentric and the geocentric. The problem with the latter is that its origin moves non linearly along a geodesic in the barycentic reference system. In this case, one has to add to $w_{m}$ of the ensemble of masses, actually limited to the Earth and its environment, and to the tidal potential $w_{e}$ that should include the Moon, the Sun, and the planets, a third quantity $w_{i}$ representing the acceleration parts due to the rotation of the geocentric reference system in the barycentric reference system. The expressions for the corrections to $w$ and to $w_{i}$ can be found in Klioner (1993). The principal effect of these additional terms is the geodetic precession, a rotation of the geocentric reference system with respect to the barycentric, whose value is 1.92 mas per year. This is a secular effect, but there are also periodic terms, the most important of which is a yearly 'geodetic nutation' with an amplitude of 0.153 mas (Fukushima, 1991) with a semiannual term of amplitude $2 \mu$ as.

Let us remark that the equivalent of the geodetic precession exists in comparing the barycentric and the galactocentric reference systems. The corresponding rate of rotation is of the order of 8.5 nas (nanoseconds of arc) per year (Brumberg, 1991b). It is much smaller than the effect of the variable part of the galactic aberration described above and is negligible.

\section{Link to measurements}

A reference system is a theoretical concept. Its realization in the form of a reference frame is the major task of astronomy. It is obviously based on various types of observations. What is the significance in the framework of general relativity of the measurements performed either from the ground or from a spacecraft? A very complete answer was given for distance, angle, and direction determinations including relativistic aberration by Brumberg (1991a) for observations within the solar system.

But it appears now that it is also necessary in some cases of astrometric or related observations to take into account the effects that take place outside the solar system for which General Relativity rather than the classical approach must be used. This is the case of radial velocity measurements. The problem is presented by Lindegren et al. (1999). The Doppler shift, even reduced to the barycentric reference system, is a result of the reduction of a measurement and is not the actual radial velocity which depends on additional factors such as transverse velocity and the gravitational potential of the source that are generally model dependent. Therefore it is necessary to clearly state that the 
relative shift in wavelengths is a measurement and not a physical quantity related to the observed body.

\section{References}

Brumberg, V.A., 1991a, Essential Relativistic Celestial Mechanics, Adam Hilger, Bristol.

Brumberg, V.A., 1991b, in Reference Systems, Proceedings of the IAU Colloquium 127, J.A. Hughes, C.A . Smith and G.H. Kaplan (eds), U.S. Naval Observatory publication, Washington, 36 .

Brumberg, V.A., 2000, in private discussions within the JCR.

Brumberg, V.A. \& Kopejkin, S.M., 1989, in Reference Frames in Astronomy and Geophysics, J. Kovalevsky, I.I. Mueller and B. Kołaczek (eds.), Kluwer Acad. Publ., 115.

Fukushima, T., 1991, Astron. Astrophys., 244, L11.

Green, R.M., 1985, Spherical astronomy, Cambridge University Press, Cambridge.

Klioner, S.A., 1993, Astron. Astrophys., 279, 273.

Klioner, S.A. \& Soffel, M., 1998, Astron. Astrophys., 334, 1123.

Kovalevsky, J. \& Mueller, I.I., 1989, in Reference Frames in Astronomy and Geophysics, J. Kovalevsky, I.I. Mueller and B. Kołaczek (eds.), Kluwer Acad. Publ., 1.

Lindegren, L., Dravins, D. \& Madsen, S., 1999, ASP Conf. Series, 185, 73.

Rindler, W., 1977, Essential Relativity, Springer Verlag, New York.

Soffel, M., 1989, Relativity in Astrometry, Celestial Mechanics and Geodesy, Springer Verlag, Berlin.

Soffel, M., Klioner, S., Petit, G. \& Wolf, P., 2000, New relativistic framework for the realization of space-time reference frames and its application to the time and frequency in the solar system, in Journées des systèmes de référence, Dresden (in press). 